\title{
Nanoantenna-Enhanced Light-Matter Interaction in Atomically Thin $\mathrm{WS}_{2}$
}

Johannes Kern, ${ }^{\dagger}$ Andreas Trügler, ${ }^{\ddagger}$ Iris Niehues, ${ }^{\dagger}$ Johannes Ewering, ${ }^{\dagger}$ Robert Schmidt, ${ }^{\dagger}$ Robert Schneider, ${ }^{\dagger}$ Sina Najmaei, ${ }^{\S}$ Antony George, ${ }^{\S}$ Jing Zhang, ${ }^{\S}$ Jun Lou, ${ }^{\S}$ Ulrich Hohenester, ${ }^{\ddagger}$ Steffen Michaelis de Vasconcellos, ${ }^{\dagger}$ and Rudolf Bratschitsch ${ }^{*}{ }^{\dagger}$

${ }^{\dagger}$ Institute of Physics and Center for Nanotechnology, University of Münster, 48149 Münster, Germany

${ }^{\ddagger}$ Institute of Physics, University of Graz, 8010 Graz, Austria

${ }^{\S}$ Department of Mechanical Engineering and Material Science, Rice University, Houston, Texas 77005, United States

\section{Supporting Information}

ABSTRACT: Atomically thin transition metal dichalcogenides (TMDCs) are an emerging class of two-dimensional semiconductors. Recently, the first optoelectronic devices featuring photodetection as well as electroluminescence have been demonstrated using monolayer TMDCs as active material. However, the light-matter coupling for atomically thin TMDCs is limited by their small absorption length and low photoluminescence

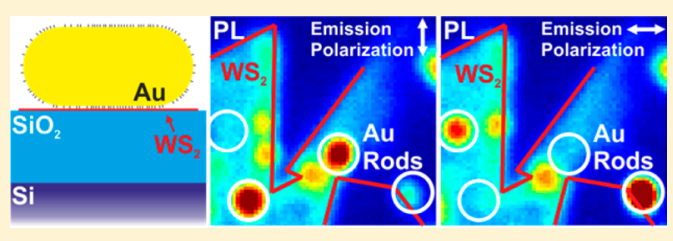
quantum yield. Here, we significantly increase the light-matter interaction in monolayer tungsten disulfide $\left(\mathrm{WS}_{2}\right)$ by coupling the atomically thin semiconductor to a plasmonic nanoantenna. Due to the plasmon resonance of the nanoantenna, strongly enhanced optical near-fields are generated within the WS $\mathrm{Wonolayer}_{2} \mathrm{We}$ observe an increase in photoluminescence intensity by more than 1 order of magnitude, resulting from a combined absorption and emission enhancement of the exciton in the $\mathrm{WS}_{2}$ monolayer. The polarization characteristics of the coupled system are governed by the nanoantenna. The robust nanoantenna-monolayer hybrid paves the way for efficient photodetectors, solar cells, and light-emitting devices based on two-dimensional materials.

KEYWORDS: plasmonics, nanoantenna, $2 \mathrm{D}$ materials, transition metal dichalcogenides, $W S_{2}$ photoluminescence, dark-field scattering

$\mathrm{T}$ ailoring the interaction between light and matter is crucial for the performance of optical and optoelectronic devices. Solar cells, photodetectors, and optical transistors rely on their ability to efficiently absorb light. Light-emitting devices benefit from a high radiative efficiency of the active material. One strategy to improve device performance is to identify novel materials that exhibit an intrinsically strong light-matter interaction. Recently, an extraordinary optical response of atomically thin transition metal dichalcogenides (TMDCs) has been reported. ${ }^{1}$ Monolayers of $\mathrm{MoS}_{2}, \mathrm{MoSe}_{2}, \mathrm{WS}_{2}$, and $\mathrm{WSe}_{2}$ exhibit an optical band gap $^{2-5}$ and are therefore interesting for optoelectronic devices such as photodetectors and lightemitting devices. ${ }^{6-11}$ However, the absorption is limited by the atomic thickness of the TMDC monolayers. Concerning light emission, atomically thin TMDCs exhibit a direct optical band gap with a photoluminescence yield that is orders of magnitude larger than for bulk crystals. ${ }^{2}$ However, in absolute numbers the observed photoluminescence quantum yield of $10^{-3}$ is low. ${ }^{2}$ Consequently, for practical device applications, strategies for increasing the light-matter interaction are needed.

Plasmonic nanoantennas made of noble metals enhance the light-matter interaction and control light on the nanoscale. $^{12-14}$ Excitation of the antenna resonance leads to intense optical fields in the vicinity of the antenna. In this region of high photonic density of states the absorption as well as the emission of photons can be significantly enhanced. By coupling to a plasmonic antenna strong enhancement of the fluorescence ${ }^{15,16}$ and Raman ${ }^{17}$ response of single molecules has been achieved. The efficiency of photodetectors and solar cells based on bulk semiconductors ${ }^{18,19}$ has been improved.

Recently, plasmonic nanostructures have been placed above and below $\mathrm{MoS}_{2}$ monolayers. ${ }^{20-25}$ Interesting phenomena such as a reversible structural phase transition in $\mathrm{MoS}_{2}$ due to hot electron injection ${ }^{23}$ or a thermally induced spectral modification of photoluminescence ${ }^{24}$ have been observed. However, so far little attention has been paid to the design of the plasmonic nanostructures. As a consequence, the reported photoluminescence enhancements were only 2 -fold at best. ${ }^{22,24}$ Even photoluminescence quenching was observed. ${ }^{21}$ Photoluminescence enhancement was attributed to an increased absorption. Here, we demonstrate an enhancement in both absorption and emission, leading to a record photoluminescence increase by 1 order of magnitude from an atomically thin semiconductor. We use a novel hybrid system consisting of a $\mathrm{WS}_{2}$ monolayer and a single-crystalline plasmonic nanoantenna. In a combined numerical and experimental study we demonstrate that in this way a hybrid plasmon-exciton system is formed. The hybrid nature is revealed by the observation of a

Received: March 13, 2015

Published: July 24, 2015 
6-fold (4-fold) PL intensity enhancement with respect to a $\mathrm{WS}_{2}$ monolayer without antenna when excitation (emission) polarization is matched to the antenna. Our analysis on single nanoantennas unambiguously shows that the coupling results from the enhanced plasmonic near-fields at the position of $\mathrm{WS}_{2}$ monolayer. We are also able to control the shape of the photoluminescence spectrum with the help of the antenna by tuning the plasmonic resonance via the antenna length. Our work provides important groundwork for the design of future nanoantenna-enhanced photodetectors, solar cells, and lightemitting devices based on two-dimensional materials.

Figure 1a shows a schematic drawing of the investigated hybrid system of a plasmonic nanoantenna and atomically thin
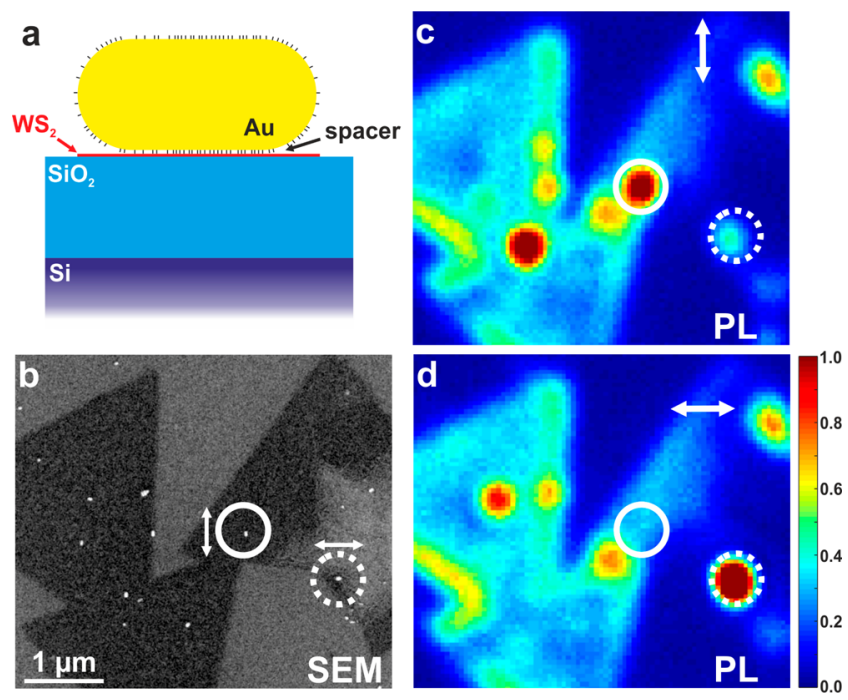

Figure 1. (a) Schematic drawing of the sample. (b) Electron micrograph of monolayer $\mathrm{WS}_{2}$ (dark triangles) with gold nanorods on top (bright rods). Solid (dashed) circles mark a rod with vertical and (horizontal) orientation. (c, d) Normalized photoluminescence intensity map of the region shown in (b). White arrows indicate the emission polarization. Strongest PL enhancement is found for orientation of the nanoantenna along the polarization direction.

$\mathrm{WS}_{2}$ layer. The $\mathrm{WS}_{2}$ monolayers are grown on a $\mathrm{SiO}_{2} / \mathrm{Si}$ substrate by chemical vapor deposition (CVD) ${ }^{26}$ using $\mathrm{WO}_{3}$ powder as a precursor and an evaporation temperature close to $800{ }^{\circ} \mathrm{C}$. The antennas are single-crystalline, chemically grown gold nanorods with a diameter of $30 \mathrm{~nm}$ and lengths ranging from 55 to $70 \mathrm{~nm}$. The rods are obtained in aqueous solution from Nanopartz Inc. and diluted to a concentration of $10^{10}$ particles/mL. A $1 \mu \mathrm{L}$ portion of the solution is drop-cast onto monolayers of tungsten disulfide $\left(\mathrm{WS}_{2}\right)$ on the $\mathrm{SiO}_{2} / \mathrm{Si}$ substrate. The gold nanorods are functionalized by a molecular layer (cetyltrimethylammonium bromide, $\mathrm{CTAB}$ ), which acts as a spacer between gold and $\mathrm{WS}_{2}$. In the electron micrograph of Figure 1 atomically thin $\mathrm{WS}_{2}$ monolayers of triangular shape appear dark. Gold nanorods on top of the monolayer are bright and can be clearly identified. Due to the drop-casting process, the gold nanorods are randomly oriented on the $\mathrm{WS}_{2}$ monolayers. In Figure $1 \mathrm{~b}$ a horizontally aligned rod is marked by a dashed white circle and a rod with vertical orientation by a solid circle (see Figure S1 for images at high magnification).

Photoluminescence intensity maps recorded for excitation with circularly polarized light at a wavelength of $\lambda_{\mathrm{ex}}=588 \mathrm{~nm}$ and selection of vertical and horizontal emission polarization are presented in Figure $1 \mathrm{c}$ and $\mathrm{d}$, respectively. Photo- luminescence emission is collected with an objective lens with a numerical aperture NA $=0.9$ and detected via a spectrometer (Andor, Shamrock SR-303i) equipped with a cooled sCMOS camera (Andor, DC-152-Q-C00_DI). The photoluminescence emission from the $\mathrm{WS}_{2}$ monolayer alone shows no polarization dependence and is homogeneous across the flake. However, stronger luminescence is typically observed at the edge of the $\mathrm{WS}_{2}$ flakes. ${ }^{27}$ At position of the gold nanoantennas the photoluminescence is strongly enhanced by a factor of 4.5 for the horizontally aligned rod and 2.5 for the vertically aligned rod. If both excitation and emission polarization are matched to the antenna, we observe an enhancement of up to 11 (Figure S2 and discussion below). For the hybrid antenna-monolayer system the photoluminescence is polarization dependent and is strongest for polarization along the long nanoantenna axis. If the polarization is perpendicular to the nanorod, the luminescence intensity is significantly lower and is similar to locations on the $\mathrm{WS}_{2}$ monolayer without gold nanorods. We also observe intrinsic gold nanorod photoluminescence. ${ }^{28}$ However, it is comparatively very weak, as evident from nanorods at sample positions without $\mathrm{WS}_{2}$ (top left corner of Figure $1 \mathrm{~b}-\mathrm{d}$ ).

In order to understand the strong PL enhancement and elucidate the nature of the coupling between the nanoantenna and the atomically thin semiconductor, we have to determine the spectral position of the plasmon and exciton resonances. The strength of the light-matter interaction of the hybrid system strongly depends on their spectral overlap. We first investigate the resonances of the constituents of the hybrid system: the nanoantenna and the $\mathrm{WS}_{2}$ monolayer.

Single-crystalline gold nanorods are efficient optical antennas and exhibit intense optical near-fields and enhanced scattering at their longitudinal plasmon resonance. ${ }^{13}$ The position of the plasmon resonance can be tuned by the length of the nanoantenna. ${ }^{13}$ The dark-field scattering spectrum of a single $65 \mathrm{~nm}$ long antenna clearly shows a pronounced longitudinal plasmon resonance at $607 \mathrm{~nm}$, as well as the weak transverse plasmon resonance at $526 \mathrm{~nm}$ (Figure 2a).

The spectral positions of the excitonic resonances of the atomically thin semiconductor are also obtained from dark-field scattering spectra from the edges of $\mathrm{WS}_{2}$ monolayers (Figure 2b). The spectra show two distinct maxima at the A and $B$ exciton wavelengths of 618 and $565 \mathrm{~nm}$. The peak positions vary from flake to flake and are in good agreement with absorption spectra (see Supporting Information Figure S3).

Our investigated hybrid system consists of monolayer $\mathrm{WS}_{2}$ and a gold nanorod of three different lengths $(55,60$, and 70 $\mathrm{nm}$ ) and a diameter of $30 \mathrm{~nm}$. The dimensions of the antenna are chosen such that the longitudinal plasmon resonance of the nanoantenna can be tuned across the A exciton resonance of the monolayer. The $\mathrm{B}$ exciton is always off-resonant to the longitudinal nanoantenna resonance and shows no polarization dependence of the photoluminescence, similar to the A exciton of the $\mathrm{WS}_{2}$ monolayer without antenna. In the measured darkfield scattering spectra (Figure 2c-e) a clear shift of the longitudinal plasmon resonance from $600 \mathrm{~nm}$ to $650 \mathrm{~nm}$ is observed, if the antenna length is increased from $55 \mathrm{~nm}$ to 70 $\mathrm{nm}$ and the emission polarization is selected along the long antenna axis. We find a prominent narrow minimum in the broad plasmon resonance in the scattering spectra of the hybrid system with the two short antennas (Figure $2 c, d$ ). The spectral position of the dip is independent of the antenna length and occurs at the wavelength of the A exciton. For the longest 


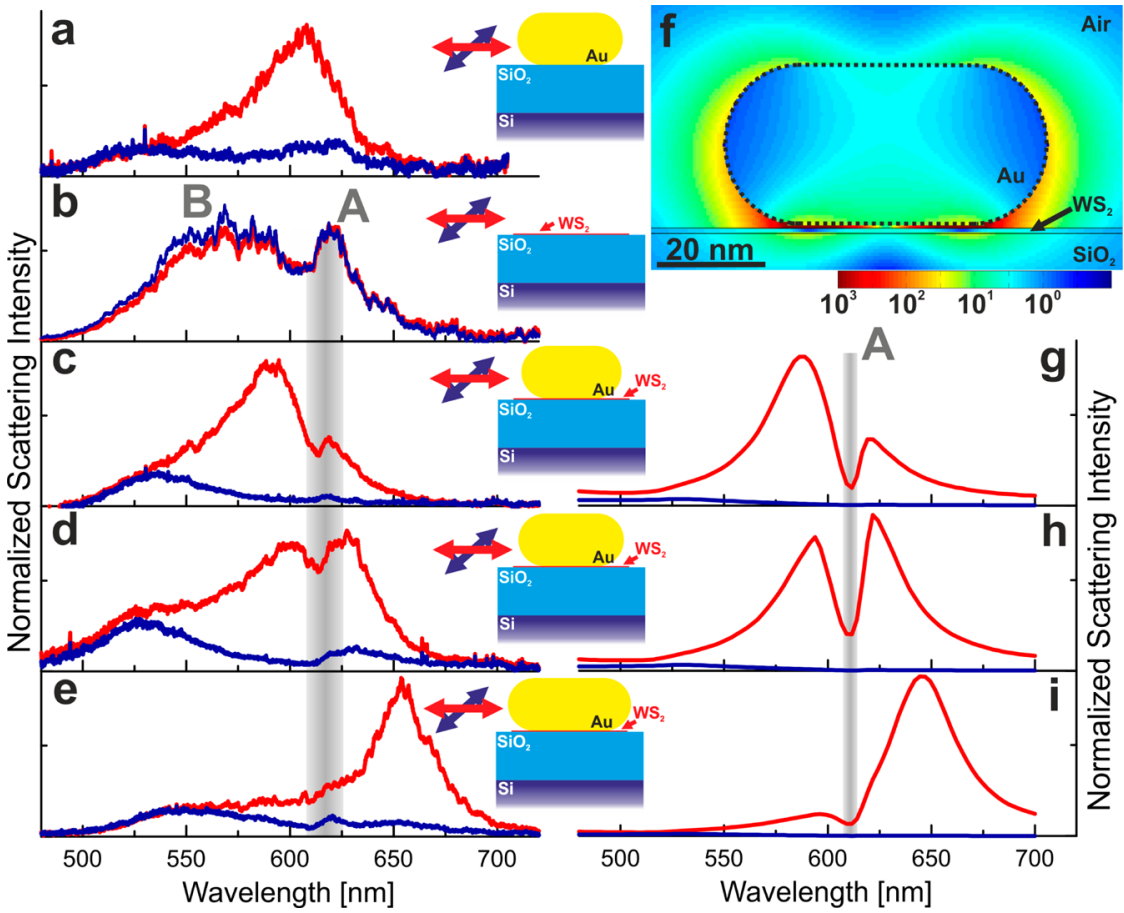

Figure 2. (a) Polarization-resolved scattering spectrum of a $65 \mathrm{~nm}$ long gold nanorod on a $\mathrm{SiO}_{2} / \mathrm{Si}$ substrate. (b) Scattering spectrum of a monolayer $\mathrm{WS}_{2}$ flake on a $\mathrm{SiO}_{2} / \mathrm{Si}$ substrate. (c-e) Measured emission-polarization-resolved scattering spectra of nanorods coupled to monolayer WS $\mathrm{W}_{2}$ on a $\mathrm{SiO}_{2} / \mathrm{Si}$ substrate. The length of the nanorods is (c) 55, (d) 60, and (e) $70 \mathrm{~nm}$. Polarization directions along/across the nanoantenna axis are indicated by colored arrows. The vertical line marks the position of the A exciton of monolayer $\mathrm{WS}_{2}$ at $(618 \pm 10) \mathrm{nm}$. (f) Calculated near-field intensity map on a logarithmic scale at $612 \mathrm{~nm}$ wavelength (A exciton) and polarization along the nanorod axis. (g-i) Calculated emissionpolarization-resolved scattering spectra of nanorods coupled to monolayer $\mathrm{WS}_{2}$ on a $\mathrm{SiO}_{2} / \mathrm{Si}$ substrate. The length of the nanorods is $(\mathrm{g}) 60,(\mathrm{~h}) 65$, and (i) $75 \mathrm{~nm}$.
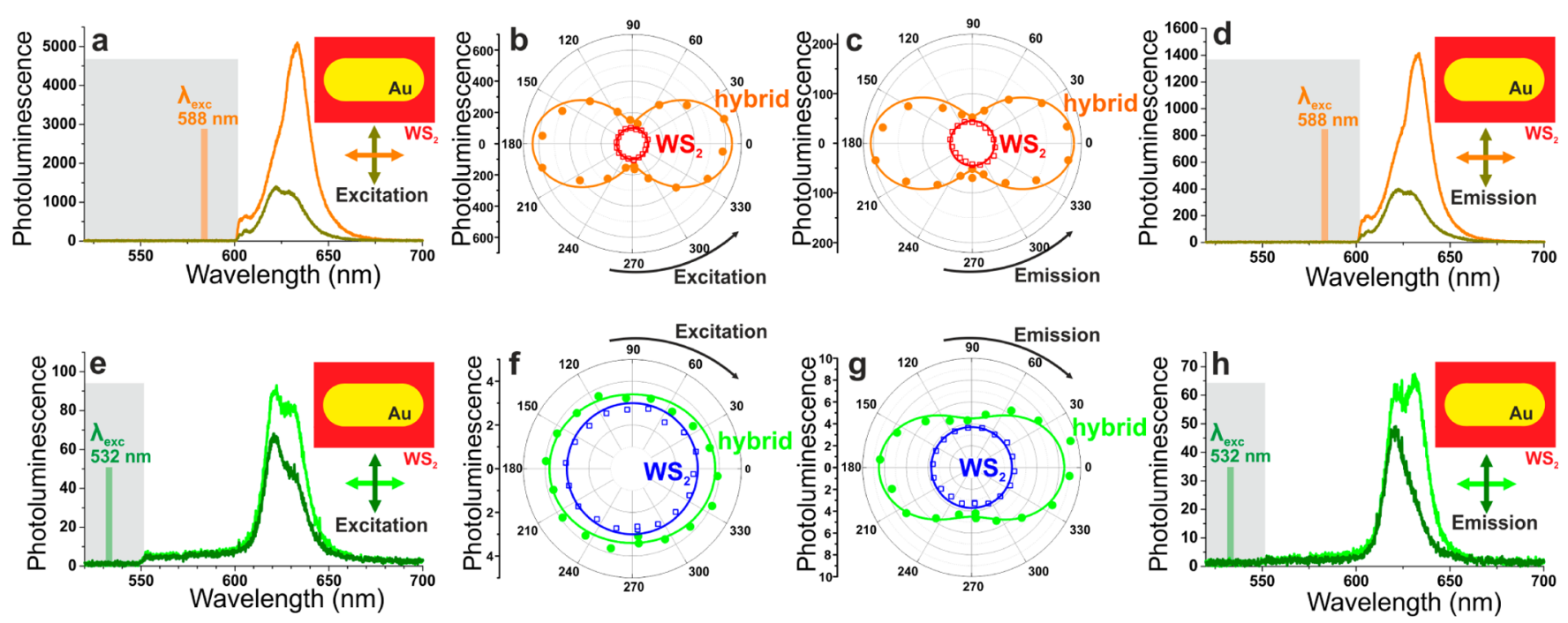

Figure 3. (a-d) PL spectrum and angular dependence for an excitation wavelength of $588 \mathrm{~nm}$. (e-h) PL spectrum and angular dependence for an excitation wavelength of $532 \mathrm{~nm}$. (a, e) PL spectrum with excitation polarization along and across the antenna. (b, f) PL intensity of a WS monolayer without nanoantenna and the nanoantenna-monolayer hybrid depending on the excitation polarization. (c, g) PL intensity of a WS monolayer without nanoantenna and the nanoantenna-monolayer hybrid depending on the emission polarization. (d, h) PL spectrum with emission polarization along and across the antenna axis for circularly polarized excitation. Gray-shaded regions in the spectra indicate band-pass filters to block light from the laser used for excitation. Solid lines drawn in the angular plots model the polarization dependence by a sum of a single horizontally orientated dipole (two-lobed) and randomly oriented dipoles (circle).

antenna $(70 \mathrm{~nm})$ there is only small spectral overlap between plasmon and exciton. Consequently, the coupling is weak and the minimum in the plasmon resonance disappears.

Our experimental results are in excellent agreement with numerically calculated scattering spectra (Figure $2 \mathrm{~g}-\mathrm{i}$ ). The simulations are performed with the MNPBEM toolbox, ${ }^{29,30}$ which uses the boundary element (BEM) approach. ${ }^{31} \mathrm{We}$ assume an antenna diameter of $30 \mathrm{~nm}$ and take a distance of 0.5 $\mathrm{nm}$ between antenna and $\mathrm{WS}_{2}$ into account (see Supplementary Figure $S 4$ for the distance dependence). In Figure $2 f$ 


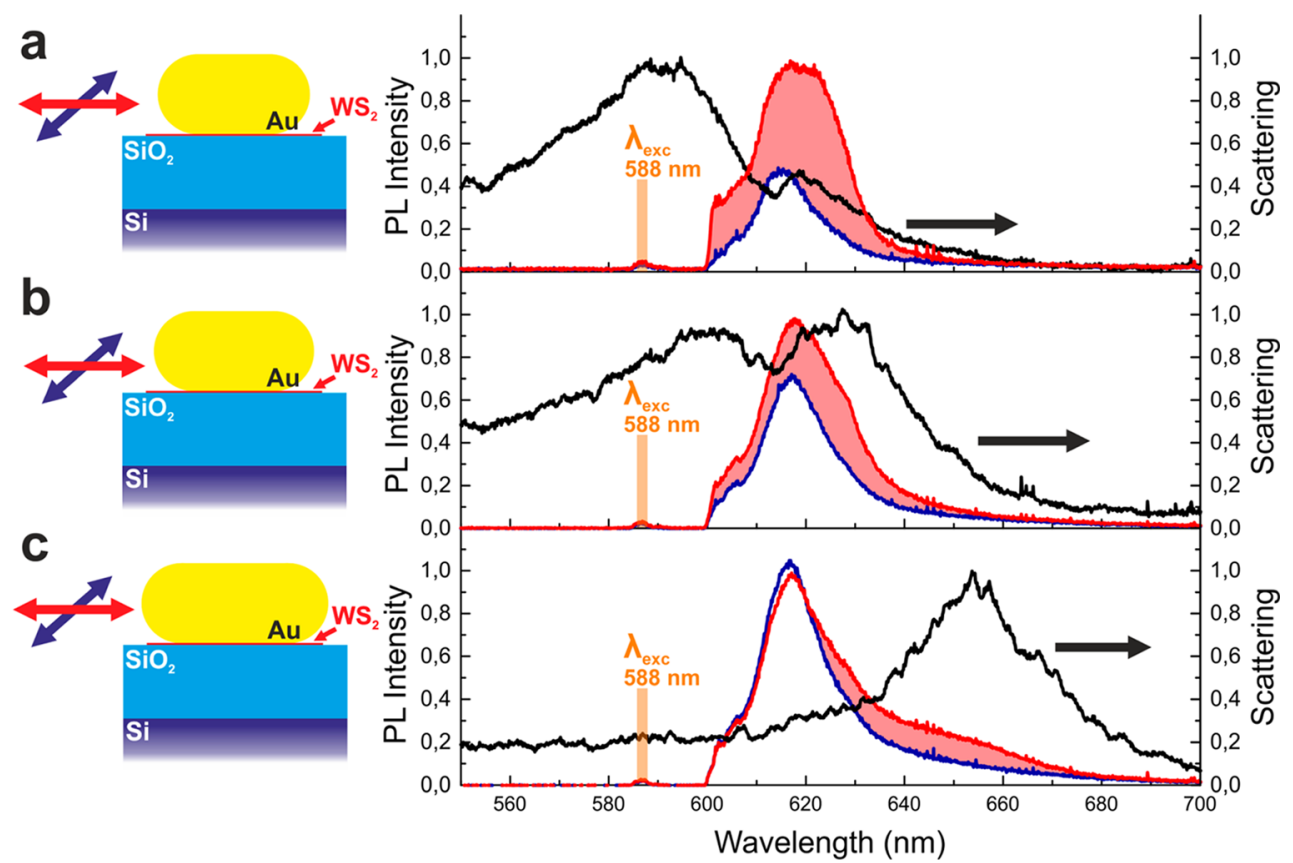

Figure 4. $(\mathrm{a}-\mathrm{c})$ Emission-polarization-resolved photoluminescence spectra (colored lines) of an antenna-WS ${ }_{2}$ hybrid compared to scattering spectra (black lines). PL spectra are excited with circular polarization with an excitation wavelength of $588 \mathrm{~nm}$. The direction of emission polarization along/across the nanoantenna long axis is indicated by arrows. The investigated antennas are the same as in Figure $2 \mathrm{c}-\mathrm{e}$, with nanorod lengths of (a) 55, (b) 60, and (c) $70 \mathrm{~nm}$.

the calculated electric field distribution for the hybrid system is shown with polarization along the long antenna axis at the wavelength of the A exciton. Intensity enhancements as high as 6 are obtained at the position of the $\mathrm{WS}_{2}$ monolayer. For polarization perpendicular to the antenna axis no enhancement is found with respect to the $\mathrm{WS}_{2}$ monolayer without antenna, and only a weak scattering signal is observed in the experiment (blue curves in Figure $2 \mathrm{c}-\mathrm{e}$ ).

In summary, we find that the A exciton of the atomically thin $\mathrm{WS}_{2}$ monolayer couples to the metal nanoantenna via the enhanced plasmonic near-field, which leads to a pronounced minimum in the scattering spectra. The strength of this dip critically depends on polarization as well as the spectral position of the plasmon with respect to the A exciton.

Having elucidated the different resonances and coupling of the hybrid system, we return to the prominent photoluminescence enhancement of Figure 1c,d. In particular, we quantify how the electric field enhancement results in an absorption and emission enhancement of the hybrid system (Figure 3). A hybrid system with a central wavelength of the plasmon resonance at $625 \mathrm{~nm}$ is chosen for this investigation, because the broad plasmon resonance overlaps well with the excitation wavelength of $588 \mathrm{~nm}$ as well as with the exciton emission at $618 \mathrm{~nm}$.

First, we study a $\mathrm{WS}_{2}$ monolayer without nanoantenna (see Supplementary Figure S5 for a photoluminescence spectrum). We find that the PL spectrum is dominated by the A exciton. ${ }^{4}$ The PL intensity does not depend on excitation or emission polarization. For excitation with $532 \mathrm{~nm}$ light the PL intensity is a factor of 2 higher than for excitation with $588 \mathrm{~nm}$. This effect is due to the different absorption (factor of 2) at the two excitation wavelengths.

The hybrid nanoantenna-monolayer system exhibits a strikingly different behavior if excited at the plasmon resonance with $588 \mathrm{~nm}$ light (Figure $3 \mathrm{a}-\mathrm{d}$ ). For excitation (Figure 3a,b) and emission polarization (Figure 3c,d) perpendicular to the nanoantenna axis the PL intensity is similar to the $\mathrm{WS}_{2}$ monolayer without nanoantenna. However, the photoluminescence strongly increases if the excitation as well as emission polarization is chosen along the nanorod axis (Figure $3 a-d$ ). This enhancement arises from the strong optical near-fields created by the longitudinal plasmon resonance of the nanoantenna, which penetrate the $\mathrm{WS}_{2}$ monolayer and extend over a region of $(80 \times 30) \mathrm{nm}^{2}$ (Figure $\left.2 \mathrm{f}\right)$. Excitons located in the enhanced electric field region experience an increased absorption as well as an increased emission rate. The latter effect results in a higher emission quantum yield of the hybrid system. In our experiment the PL enhancement is clearly observed by comparing the polarization-resolved PL of the hybrid nanoantenna-monolayer system to the $\mathrm{WS}_{2}$ monolayer alone (Figure $3 \mathrm{~b}$ for excitation and $3 \mathrm{c}$ for emission). Whereas the photoluminescence of the $\mathrm{WS}_{2}$ monolayer is unpolarized (circle), a clear dipolar-like pattern is observed for excitation and emission polarization of the hybrid system. To study the emission polarization, the system was excited by circularly polarized light. The PL signal of the hybrid system is a factor of 6.4 (4.5) larger compared to the PL of the monolayer alone when the excitation (emission) polarization is matched to the antenna axis. These high values underline the increased coupling of the nanoantenna with the atomically thin semiconductor. The fact that absorption enhancement plays a more important role than emission enhancement is revealed by investigating the identical hybrid system with off-resonant excitation at a wavelength of $532 \mathrm{~nm}$. In this case the PL intensity is more than 10 times lower that for resonant excitation at $588 \mathrm{~nm}$. If the excitation polarization is perpendicular to the long antenna axis, the PL intensity resembles that of a $\mathrm{WS}_{2}$ monolayer without nanoantenna. For excitation polarization along the antenna axis the $\mathrm{PL}$ is increased by a factor of 1.3 (Figure 3e,f). Even though the 
excitation is off-resonant, the plasmon and exciton resonances still overlap, and the emission rate is expected to increase. Indeed, the PL signal is 2.3 times stronger for emission polarization along the antenna axis (Figure 3g,h).

Considering the fact that the spatial extension of the enhanced optical near-fields of the nanoantenna penetrating the $\mathrm{WS}_{2}$ monolayer is a factor of 100 smaller than the diffraction-limited collection area of PL light, the local PL enhancement is estimated to be 1000 . Moreover, it should be noted that even the highest applied continuous wave excitation power density of $14 \mathrm{~kW} / \mathrm{cm}^{2}$ is well below saturation of this hybrid system (Supplementary Figure S6). Much stronger enhancement can be expected for even higher excitation powers, where the saturation of the $\mathrm{WS}_{2}$ monolayer can be compensated by an increased emission rate.

It is expected that an increased emission rate due to the antenna resonance not only enhances the PL intensity but also modifies the PL spectrum. ${ }^{32}$ To this end we record emissionpolarization-resolved photoluminescence spectra for excitation with circular polarization with an excitation wavelength of 588 $\mathrm{nm}$ (Figure 4). We investigate three antenna-WS 2 hybrids with previously characterized plasmon resonance (Figure $2 c-e$ ). For emission polarization set perpendicular to the nanoantenna axis the photoluminescence spectrum resembles that of a $\mathrm{WS}_{2}$ monolayer. However, for emission polarization along the antenna axis the spectrum is enhanced at the plasmon resonance. The modification strongly depends on the length of the antenna, which further demonstrates the coupling of exciton and plasmon. The fact that spectral modification of the $\mathrm{PL}$ is observed only for emission polarization along the antenna suggests that it originates from an enhanced emission rate, and we can exclude thermal effects ${ }^{24}$ as well as morphology changes $^{23}$ of the $\mathrm{WS}_{2}$ monolayers.

It should be noted that we have observed robust photoluminescence of the hybrid nanoantenna-monolayer systems over a time period of several months, which allowed us to conduct systematic studies on a single nanostructure. This behavior is in stark contrast to earlier experiments of antennaenhanced fluorescence of single molecules, where enhanced fluorescence was observed only for a few $100 \mathrm{~ms}$ before photobleaching occurred. ${ }^{16}$

In conclusion, we have demonstrated antenna-enhanced light-matter coupling in atomically thin $\mathrm{WS}_{2}$. Due to intense optical near-fields provided by the metal nanoantenna, we observe an absorption as well as emission enhancement, resulting in a 1 order of magnitude increase of the photoluminescence of the $\mathrm{WS}_{2}$ monolayer. We find that the polarization characteristics as well as the photoluminescence spectrum are modified by the longitudinal plasmon resonance. The robust hybrid nanoantenna-monolayer system lights the way to efficient photodetectors, solar cells, and light-emitting and conceptually new valleytronic devices based on twodimensional materials.

Note added: During the review process we became aware of a related study ${ }^{33}$ of $\mathrm{Ag}$ nanodisc arrays fabricated by electronbeam lithography on monolayer $\mathrm{MoS}_{2}$.

\section{ASSOCIATED CONTENT}

\section{S Supporting Information}

The Supporting Information is available free of charge on the ACS Publications website at DOI: 10.1021/acsphotonics.5b00123.
Photoluminescence enhancement of monolayer $\mathrm{WS}_{2}$ with both excitation and emission polarization matched to the nanoantenna; absorption spectrum of a $\mathrm{WS}_{2}$ monolayer; numerically calculated scattering spectra of the antenna- $\mathrm{WS}_{2}$ hybrid as a function of the distance between antenna and $\mathrm{WS}_{2}$; photoluminescence of the $\mathrm{WS}_{2}$ monolayer without nanoantenna; photoluminescence intensity depending on excitation power; details of the simulation technique (PDF)

\section{AUTHOR INFORMATION}

\section{Corresponding Author}

*E-mail: Rudolf.Bratschitsch@uni-muenster.de.

\section{Notes}

The authors declare no competing financial interest.

\section{ACKNOWLEDGMENTS}

We gratefully acknowledge financial support by the Deutsche Forschungsgemeinschaft (SPP 1391) and the Welch Foundation (grant C-1716).

\section{REFERENCES}

(1) Wang, Q. H.; Kalantar-Zadeh, K.; Kis, A.; Coleman, J. N.; Strano, M. S. Electronics and Optoelectronics of Two-Dimensional Transition Metal Dichalcogenides. Nat. Nanotechnol. 2012, 7, 699-712.

(2) Mak, K. F.; Lee, C.; Hone, J.; Shan, J.; Heinz, T. F. Atomically Thin $\mathrm{MoS}_{2}$ : A New Direct-Gap Semiconductor. Phys. Rev. Lett. 2010, $105,136805$.

(3) Splendiani, A.; Sun, L.; Zhang, Y.; Li, T.; Kim, J.; Chim, C.-Y.; Galli, G.; Wang, F. Emerging Photoluminescence in Monolayer $\mathrm{MoS}_{2}$. Nano Lett. 2010, 10, 1271-1275.

(4) Zhao, W.; Ghorannevis, Z.; Chu, L.; Toh, M.; Kloc, C.; Tan, P.H.; Eda, G. Evolution of Electronic Structure in Atomically Thin Sheets of $\mathrm{WS}_{2}$ and $\mathrm{WSe}_{2}$. ACS Nano 2013, 7, 791-797.

(5) Tonndorf, P.; Schmidt, R.; Böttger, P.; Zhang, X.; Börner, J.; Liebig, A.; Albrecht, M.; Kloc, C.; Gordan, O.; Zahn, D. R.; et al. Photoluminescence Emission and Raman Response of Monolayer $\mathrm{MoS}_{2}, \mathrm{MoSe}_{2}$, and $\mathrm{WSe}_{2}$. Opt. Express 2013, 21, 4908-4916.

(6) Lopez-Sanchez, O.; Lembke, D.; Kayci, M.; Radenovic, A.; Kis, A. Ultrasensitive Photodetectors Based on Monolayer $\mathrm{MoS}_{2}$. Nat. Nanotechnol. 2013, 8, 497-501.

(7) Yin, Z.; Li, H.; Li, H.; Jiang, L.; Shi, Y.; Sun, Y.; Lu, G.; Zhang, Q.; Chen, X.; Zhang, H. Single-Layer $\mathrm{MoS}_{2}$ Phototransistors. ACS Nano 2012, 6, 74-80.

(8) Pospischil, A.; Furchi, M. M.; Mueller, T. Solar-Energy Conversion and Light Emission in an Atomic Monolayer P-N Diode. Nat. Nanotechnol. 2014, 9, 257-261.

(9) Baugher, B. W. H.; Churchill, H. O. H.; Yang, Y.; Jarillo-Herrero, P. Optoelectronic Devices Based on Electrically Tunable P-N Diodes in a Monolayer Dichalcogenide. Nat. Nanotechnol. 2014, 9, 262-267.

(10) Ross, J. S.; Klement, P.; Jones, A. M.; Ghimire, N. J.; Yan, J.; Mandrus, D. G.; Taniguchi, T.; Watanabe, K.; Kitamura, K.; Yao, W.; et al. Electrically Tunable Excitonic Light-Emitting Diodes Based on Monolayer WSe ${ }_{2}$ P-N Junctions. Nat. Nanotechnol. 2014, 9, 268-272.

(11) Lee, C.-H.; Lee, G.-H.; van der Zande, A. M.; Chen, W.; Li, Y.; Han, M.; Cui, X.; Arefe, G.; Nuckolls, C.; Heinz, T. F.; et al. Atomically Thin $\mathrm{P}-\mathrm{N}$ Junctions with van Der Waals Heterointerfaces. Nat. Nanotechnol. 2014, 9, 676-681.

(12) Novotny, L.; van Hulst, N. Antennas for Light. Nat. Photonics 2011, 5, 83-90.

(13) Biagioni, P.; Huang, J. S.; Hecht, B. Nanoantennas for Visible and Infrared Radiation. Rep. Prog. Phys. 2012, 75, 024402.

(14) Merlein, J.; Kahl, M.; Zuschlag, A.; Sell, A.; Halm, A.; Boneberg, J.; Leiderer, P.; Leitenstorfer, A.; Bratschitsch, R. Nanomechanical Control of an Optical Antenna. Nat. Photonics 2008, 2, 230-233. 
(15) Kinkhabwala, A.; Yu, Z.; Fan, S.; Avlasevich, Y.; Müllen, K.; Moerner, W. E. Large Single-Molecule Fluorescence Enhancements Produced by a Bowtie Nanoantenna. Nat. Photonics 2009, 3, 654-657.

(16) Khatua, S.; Paulo, P. M. R.; Yuan, H.; Gupta, A.; Zijlstra, P.; Orrit, M. Resonant Plasmonic Enhancement of Single-Molecule Fluorescence by Individual Gold Nanorods. ACS Nano 2014, 8, 4440-4449.

(17) Nie, S. Probing Single Molecules and Single Nanoparticles by Surface-Enhanced Raman Scattering. Science 1997, 275, 1102-1106.

(18) Knight, M. W.; Sobhani, H.; Nordlander, P.; Halas, N. J. Photodetection with Active Optical Antennas. Science 2011, 332, 702704.

(19) Atwater, H. A.; Polman, A. Plasmonics for Improved Photovoltaic Devices. Nat. Mater. 2010, 9, 205-213.

(20) Lin, J.; Li, H.; Zhang, H.; Chen, W. Plasmonic Enhancement of Photocurrent in $\mathrm{MoS}_{2}$ Field-Effect-Transistor. Appl. Phys. Lett. 2013, 102, 203109.

(21) Bhanu, U.; Islam, M. R.; Tetard, L.; Khondaker, S. I. Photoluminescence Quenching in Gold $\mathrm{MoS}_{2}$ Hybrid Nanoflakes. Sci. Rep. 2014, 4, 5575.

(22) Sobhani, A.; Lauchner, A.; Najmaei, S.; Ayala-Orozco, C.; Wen, F.; Lou, J.; Halas, N. J. Enhancing the Photocurrent and Photoluminescence of Single Crystal Monolayer $\mathrm{MoS}_{2}$ with Resonant Plasmonic Nanoshells. Appl. Phys. Lett. 2014, 104, 031112.

(23) Kang, Y.; Najmaei, S.; Liu, Z.; Bao, Y.; Wang, Y.; Zhu, X.; Halas, N. J.; Nordlander, P.; Ajayan, P. M.; Lou, J.; et al. Plasmonic Hot Electron Induced Structural Phase Transition in a $\mathrm{MoS}_{2}$ Monolayer. Adv. Mater. 2014, 26, 6467-6471.

(24) Najmaei, S.; Mlayah, A.; Arbouet, A.; Girard, C.; Léotin, J.; Lou, J. Plasmonic Pumping of Excitonic Photoluminescence in Hybrid $\mathrm{MoS}_{2}-\mathrm{Au}$ Nanostructures. ACS Nano 2014, 8, 12682-12689.

(25) Goodfellow, K. M.; Beams, R.; Chakraborty, C.; Novotny, L.; Vamivakas, A. N. Integrated Nanophotonics Based on Nanowire Plasmons and Atomically Thin Material. Optica 2014, 1, 149-152.

(26) Najmaei, S.; Liu, Z.; Zhou, W.; Zou, X.; Shi, G.; Lei, S.; Yakobson, B. I.; Idrobo, J.-C.; Ajayan, P. M.; Lou, J. Vapour Phase Growth and Grain Boundary Structure of Molybdenum Disulphide Atomic Layers. Nat. Mater. 2013, 12, 754-759.

(27) Gutiérrez, H. R.; Perea-López, N.; Elías, A. L.; Berkdemir, A.; Wang, B.; Lv, R.; López-Urías, F.; Crespi, V. H.; Terrones, H.; Terrones, M. Extraordinary Room-Temperature Photoluminescence in Triangular WS $\mathrm{WS}_{2}$ Monolayers. Nano Lett. 2013, 13, 3447-3454.

(28) Tcherniak, A.; Dominguez-Medina, S.; Chang, W.-S.; Swanglap, P.; Slaughter, L. S.; Landes, C. F.; Link, S. One-Photon Plasmon Luminescence and Its Application to Correlation Spectroscopy as a Probe for Rotational and Translational Dynamics of Gold Nanorods. J. Phys. Chem. C 2011, 115, 15938-15949.

(29) Hohenester, U.; Trügler, A. MNPBEM A Matlab Toolbox for the Simulation of Plasmonic Nanoparticles. Comput. Phys. Commun. 2012, 183, 370-381.

(30) Waxenegger, J.; Trügler, A.; Hohenester, U. Plasmonics simulations with the MNPBEM toolbox: Consideration of substrates and layer structures. Comput. Phys. Commun. 2015, 138, 193.

(31) García de Abajo, F.; Howie, A. Retarded Field Calculation of Electron Energy Loss in Inhomogeneous Dielectrics. Phys. Rev. B: Condens. Matter Mater. Phys. 2002, 65, 115418.

(32) Ringler, M.; Schwemer, A.; Wunderlich, M.; Nichtl, A.; Kürzinger, K.; Klar, T.; Feldmann, J. Shaping Emission Spectra of Fluorescent Molecules with Single Plasmonic Nanoresonators. Phys. Rev. Lett. 2008, 100, 203002.

(33) Butun, S.; Tongay, S.; Aydin, K. Enhanced Light Emission from Large-Area Monolayer $\mathrm{MoS}_{2}$ Using Plasmonic Nanodisc Arrays. Nano Lett. 2015, 15 (4), 2700-2704. 